\title{
An IgG4-Related Salivary Gland Disorder: A Case Series Presenting with a Different Clinical Setting
}

\author{
Masayuki Ishida, Hiroaki Fushiki, and Yukio Watanabe \\ Department of Otolaryngology, Head \& Neck Surgery, University of Toyama, Toyama 930-0194, Japan \\ Correspondence should be addressed to Hiroaki Fushiki, hfushiki@med.u-toyama.ac.jp
}

Received 1 June 2011; Accepted 28 June 2011

Academic Editors: F. Blanco-Favela, A. Plebani, M.-Y. Shiau, and P. Szodoray

Copyright ( $) 2011$ Masayuki Ishida et al. This is an open access article distributed under the Creative Commons Attribution License, which permits unrestricted use, distribution, and reproduction in any medium, provided the original work is properly cited.

\begin{abstract}
Küttner tumor is a chronic inflammatory disease that presents with a firm swelling of the submandibular gland and often mimics a neoplasm. Recently evidence suggests that Küttner tumor may be a type of disorder characterized by IgG4-related inflammations. Herein, we report 3 cases of submandibular gland swellings with severe fibrosis, inflammation with marked lymphoplasmacytic infiltration; this pathology mimics clinical manifestation of a malignant tumor in 18-fluorodeoxyglucose positron emission tomography (FDG-PET) findings.
\end{abstract}

\section{Introduction}

Küttner tumor, commonly known as chronic sclerosing sialadenitis, is a chronic inflammatory disease that presents with firm swelling of the submandibular gland and often mimics a malignant tumor clinically [1]. Recent studies have shown that the pathogenesis of Küttner tumor can be characterized by IgG4-related multifocal inflammations $[2$, $3]$. In the present study, we report 3 cases of an IgG4-related salivary gland disorder that cause bilateral or unilateral swelling of the submandibular gland, each presenting with a different clinical setting. Diagnostic features of the disorder will be discussed together with a literature review.

\section{Case Report}

Case 1. A 77-year-old male visited our university hospital because of a 2-month history of bilateral swelling of the submandibular region. Bilateral diffused enlargement of the submandibular glands was elastic hard and had good mobility on palpation. A computed tomography (CT) scan showed that the submandibular gland was bilaterally enlarged to approximately $30 \mathrm{~mm}$ (Figure 1). There was no significant swelling of lymph nodes in the neck. Laboratory tests were negative for SS-A and SS-B antibodies, with no increase in amylase activity. The swelling could not be resolved by treatment with antibiotics and antiphlogistic agents. A biopsy sample of the left submandibular gland was taken under local anesthesia. The biopsied tissue was white and hard. Histopathological examination revealed extensive fibrosis and diffuse lymphocyte infiltration, with scattering of a small number of eosinophilic leucocytes and lymphoid follicles. The salivary gland tissue was highly atrophied.

Case 2. A 59-year-old female was admitted to our university hospital because of a swelling in the right submandibular gland region for the third time. Swellings in the right submandibular gland region had occurred twice in the previous 2 years, and the biopsy samples showed "reactive lymph node" both times. CT showed that the right submandibular gland was enlarged to $40 \mathrm{~mm} \times 20 \mathrm{~mm}$, with several lymph nodes measuring up to $15 \mathrm{~mm}$ in the periphery of the parotid gland and the right upper deep cervical region.

18-fluorodeoxyglucose positron emission tomography (FDG-PET) showed a high and slightly heterogeneous increase in accumulations of FDG in the right submandibular region (standardized uptake value, SUV: 6.24), with a slight increase in accumulations in the upper dorsal to the right submandibular region, the right cervical regions, and the supraclavicular fossa (Figure 2). Laboratory tests were negative for SS-A and SS-B antibodies, with no increase in amylase activity. In order to rule out a possible right 


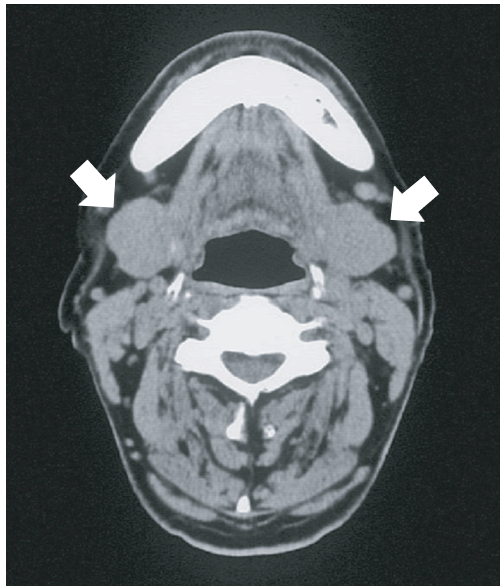

Figure 1: Cervical CT images of Case 1. Bilateral swelling of the submandibular glands (arrows).

submandibular gland cancer with lymph node metastases or malignant lymphoma, the right submandibular gland was extirpated under general anesthesia. Histopathological examination revealed diffused, double-layered, small-sized gland ducts accompanied by lymphoid follicles forming a high-degree lymphocyte infiltration. Plasma cell and eosinophilic leukocyte infiltrations, relatively high-degree fibrosis, and many IgG4-positive plasma cells were also observed (Figure 3). Subsequent swelling occurred in the unaffected submandibular gland after a period, which was resolved by steroid treatment.

Case 3. A 61-year-old male was admitted to our university hospital because of a 1-year history of swelling of the right submandibular region. He reported that the swelling enlarged gradually over the year. CT showed a slightly contrast-enhanced mass, measuring $32 \mathrm{~mm} \times 22 \mathrm{~mm}$ in the right submandibular region. Salivary gland scintigraphy (Tc-99 $\mathrm{m}$ ) showed a decrease in accumulations in the right posterior submandibular gland in early images. Laboratory tests were negative for SS-A and SS-B antibodies, with no increase in amylase activity. The right submandibular gland was extirpated under general anesthesia. Histopathological examination revealed that the submandibular glands were enlarged because of marked lymphocyte infiltration and only a few normal submandibular glands were present. There was a remarkably high degree of plasma cell infiltration in the fibroid portion of the glands.

\section{Discussion}

In 1896, Küttner identified a disorder that causes tumorlike swelling of the submandibular glands, which was named after him [1]. It differs from nonspecific chronic sialadenitis in that the submandibular gland is commonly affected, hard tumor-like mass is present in clinical manifestation, and pathology shows florid lymphoplasmacytic infiltrate accompanied by prominent and progressive fibrosis. In 108 cases described by Isacsson and Lundquist, the mean age at diagnosis was $42-45$ years, with no gender difference [4]. The chief complaint is recurrent pain and/or swelling in one side of the neck [4]. Recent studies have shown that Küttner tumor and Mikulicz's disease are serologically similar disorders that cause IgG4-related inflammations of the salivary gland and they both may produce similar lesions in organs other than the salivary gland [2, 3, 5-7]. Lesions of IgG4-related inflammations include the lacrimal glands, autoimmune pancreatitis, sclerosing cholangitis, interstitial nephritis, and retroperitoneal fibrosis $[6,7]$.

The 3 cases reported in this study are summarized in Table 1. A definite diagnosis of the disorders in this study was made by histopathological examination of the salivary glands; all cases were diagnosed with IgG4-related chronic sclerosing sialadenitis, on the basis of the presence of high-degree infiltration of IgG4-positive plasma cells in the swollen submandibular gland tissues.

All 3 cases showed swelling of the submandibular region, but each presented with a different clinical manifestation. If the disease affects both sides, as in Case 1, systemic autoimmune disorders such as Küttner tumor, Mikulicz's disease, or Sjögren's syndrome should be considered initially in the differential diagnosis. Mikulicz's disease, first reported by Mikulicz in 1892, is a disorder of unknown cause, showing sustained left-right symmetric swellings of the lacrimal gland as well as the salivary gland [8]. In contrast, Sjögren's syndrome is manifested by xerostomia and keratoconjunctivitis sicca accompanied by the swelling of the salivary glands. Küttner tumor and Mikulicz's disease are serologically characterized by high levels of serum IgG and its subtype IgG4, and it is mostly negative for the antinuclear antibodies (ANA) and anti-SS-A and -SS-B, which are often positive in Sjögren's syndrome [5]. Histological examination showed the presence of IgG4-positive plasma cell infiltration in Mikulicz's disease but not in Sjögren's syndrome [5]. Chronic sclerosing sialadenitis with widespread involvement of the major and minor salivary glands has been reported as a usual presentation [9]. Mikulicz's disease and Küttner tumor may be subtypes of an identical clinical entity with IgG4-related inflammations, with the extent and severity of the lesions within the salivary gland presenting with different clinical manifestations.

In unilateral cases similar to our tumor-like Cases 2 and 3 , it may not be easy to diagnose this disorder. If the submandibular swelling occurs unilaterally with abnormal accumulations on FDG-PET (Case 2), malignancy such as a cancer or lymphoma is more likely to be suspected. Measurement of serum IgG4 concentrations is useful for diagnosing Mikulicz's disease: it was significantly greater in Mikulicz's disease than in other salivary gland diseases (a cutoff value of $135 \mathrm{mg} / \mathrm{dL}$ ) [10]. It may be also useful in differentiating clinically Küttner tumor from neoplasm. If serum IgG4 concentration is within normal range, biopsy sample should be taken for a definite diagnosis (Case 3). Once a definite diagnosis of IgG 4-related chronic sclerosing sialadenitis is made on the basis of pathology, FDG-PET may be useful for characterizing multifocal lesions other 


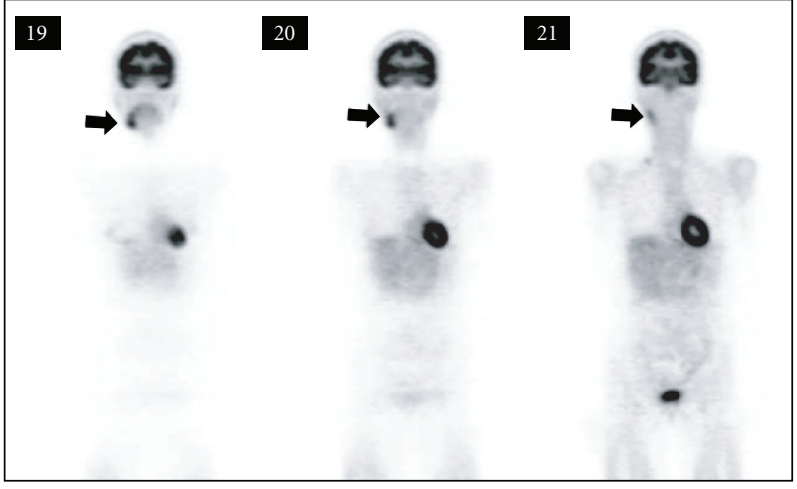

(a)

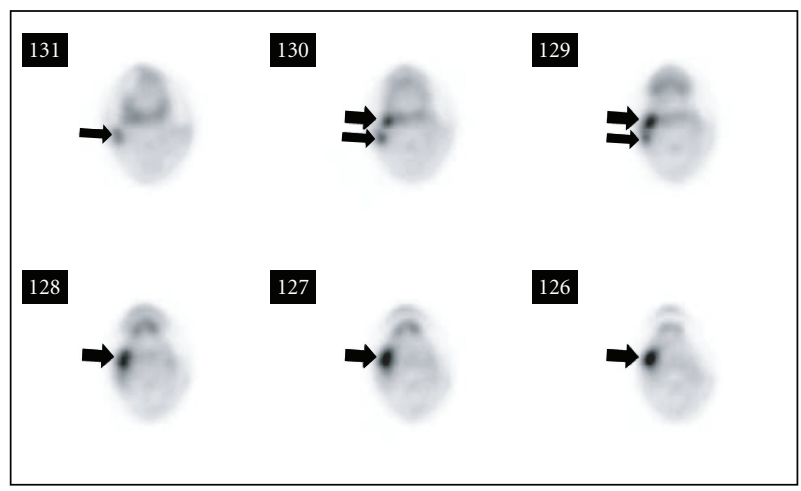

(b)

FIGURE 2: FDG-PET images of Case 2. (a) Intense uptake (SUV: 6.24) in the right submandibular gland (arrows). (b) Intense uptake in the right submandibular gland (thick arrows) and the deep interior cervical area (thin arrows).

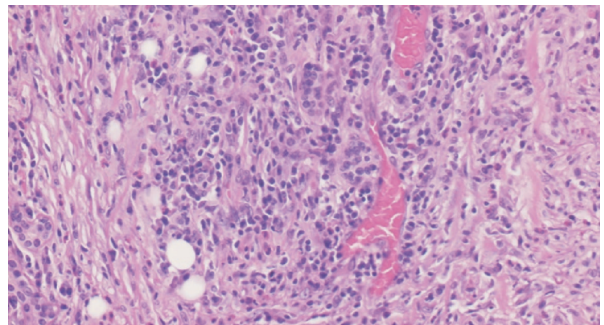

(a)

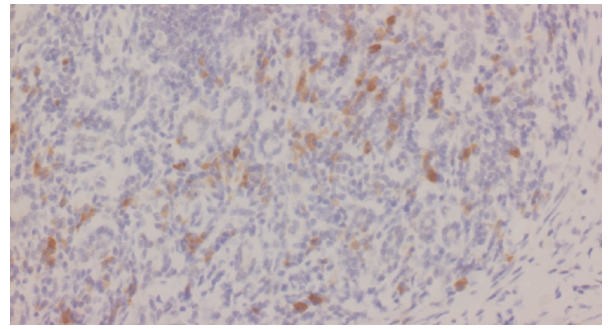

(b)

Figure 3: Histopathological examination of Case 2. (a) Small-sized gland ducts with plasma cell and eosinophilic leukocyte infiltrations (H\&E stain $\times 40)$. (b) IgG4-positive plasma cells (stained with IgG4 monoclonal antibody $\times 40$ ). Mouse monoclonal antibody to human IgG4 (clone, HP6025; dilution, 1 : 100; Zymed Laboratories Inc., Calif, USA).

TABLE 1: Summary of three case reports.

\begin{tabular}{|c|c|c|c|}
\hline & Case 1 & Case 2 & Case 3 \\
\hline Age, gender & 77 years old, male & 59 years old, female & 61 years old, male \\
\hline Swelling of the submandibular gland & Both sides & $\begin{array}{l}\text { Initially, on the right side; after a } \\
\text { period of time, on the left side }\end{array}$ & Right side \\
\hline Serum amylase & Within normal range & Within normal range & Within normal range \\
\hline Serum SS-A antibody & Negative & Negative & Negative \\
\hline Serum SS-B antibody & Negative & Negative & Negative \\
\hline $\begin{array}{l}\text { Serum IgG } \\
(863-1589 \mathrm{mg} / \mathrm{dL})\end{array}$ & 2441 & 1313 & 1196 \\
\hline $\begin{array}{l}\text { Serum IgG4 } \\
(4-108 \mathrm{mg} / \mathrm{dL})\end{array}$ & 451 & 241 & 64 \\
\hline $\begin{array}{l}\text { IgG4-positive plasma cells in salivary } \\
\text { gland tissue }\end{array}$ & Positive & Positive & Positive \\
\hline $\begin{array}{l}\text { Histopathological } \\
\text { diagnosis }\end{array}$ & Chronic sclerosing sialadenitis & Chronic sclerosing sialadenitis & $\begin{array}{l}\text { Chronic sclerosing } \\
\text { sialadenitis }\end{array}$ \\
\hline Other organ lesions & No & No & No \\
\hline Other image findings & none & Intense uptake on PET & $\begin{array}{c}\text { Decrease in accumulations } \\
\text { on salivary gland } \\
\text { scintigraphy }\end{array}$ \\
\hline
\end{tabular}


than the salivary gland and/or assessing the therapeutic effects of corticosteroids [7]. Kitagawa et al. [2] found that 5 out of 12 patients with Küttner tumor were associated with sclerosing lesions in the extrasalivary gland tissues. Treatment with steroids shows a good response in Küttner tumor and Mikulicz's disease $[3,5,11]$.

In clinical practice, we recommend that Küttner tumor should be treated as a systemic disorder rather than a salivary gland disorder, by otolaryngologists. Clinical whole-body examination and followup is necessary to find simultaneous and/or emerging IgG4-related inflammations of other sites when they did it.

\section{References}

[1] H. Küttner, "Über entzündliche tumoren der submaxillarspeicheldrüse," Beitr Klin Chir, vol. 15, pp. 815-828, 1896.

[2] S. Kitagawa, Y. Zen, K. Harada et al., "Abundant IgG4positive plasma cell infiltration characterizes chronic sclerosing sialadenitis (Küttner's tumor)," American Journal of Surgical Pathology, vol. 29, no. 6, pp. 783-791, 2005.

[3] T. Kamisawa, H. Nakajima, and T. Hishima, "Close correlation between chronic sclerosing sialadenitis and immunoglobulin G4," Internal Medicine Journal, vol. 36, no. 8, pp. 527-529, 2006.

[4] G. Isacsson, B. Ahlner, and P. G. Lundquist, "Chronic sialadenitis of the submandibular gland. A retrospective study of 108 cases," Archives of Oto-Rhino-Laryngology, vol. 232, no. 1, pp. 91-100, 1981.

[5] M. Yamamoto, C. Suzuki, Y. Naishiro, H. Takahashi, Y. Shinomura, and K. Imai, "The significance of disease-independence in Mikulicz's disease-revival interests in Mikulicz's disease," Nihon Rinshō Men'eki Gakkai Kaishi, vol. 29, no. 1, pp. 1-7, 2006 (Japanese).

[6] H. Hamano, S. Kawa, Y. Ochi et al., "Hydronephrosis associated with retroperitoneal fibrosis and sclerosing pancreatitis," Lancet, vol. 359, no. 9315, pp. 1403-1404, 2002.

[7] T. Tanabe, K. Tsushima, M. Yasuo et al., "IgG4-associated multifocal systemic fibrosis complicating sclerosing sialadenitis, hypophysitis, and retroperitoneal fibrosis, but lacking pancreatic involvement," Internal Medicine, vol. 45, no. 21, pp. 1243-1247, 2006.

[8] J. Mikulicz, "Über eine eigenartige symmetrische Erkrankung der Tränen und Mundspeicheldrüsen," in Beiträge zur Chirurgie : Festschrift gewidmet Theodor Billroth, pp. 610-630, 1892.

[9] M. Blanco, T. Mesko, M. Cura, and B. Cabello-Inchausti, "Chronic sclerosing sialadenitis (Kuttner's tumor): unusual presentation with bilateral involvement of major and minor salivary glands," Annals of Diagnostic Pathology, vol. 7, no. 1, pp. 25-30, 2003.

[10] T. Tabata, T. Kamisawa, K. Takuma et al., "Serum IgG4 concentrations and IgG4-related sclerosing disease," Clinica Chimica Acta, vol. 408, no. 1-2, pp. 25-28, 2009.

[11] M. Yamamoto, S. Harada, M. Ohara et al., "Beneficial effects of steroid therapy for Mikulicz's disease," Rheumatology, vol. 44, no. 10, pp. 1322-1323, 2005. 


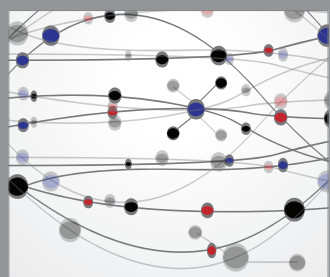

The Scientific World Journal
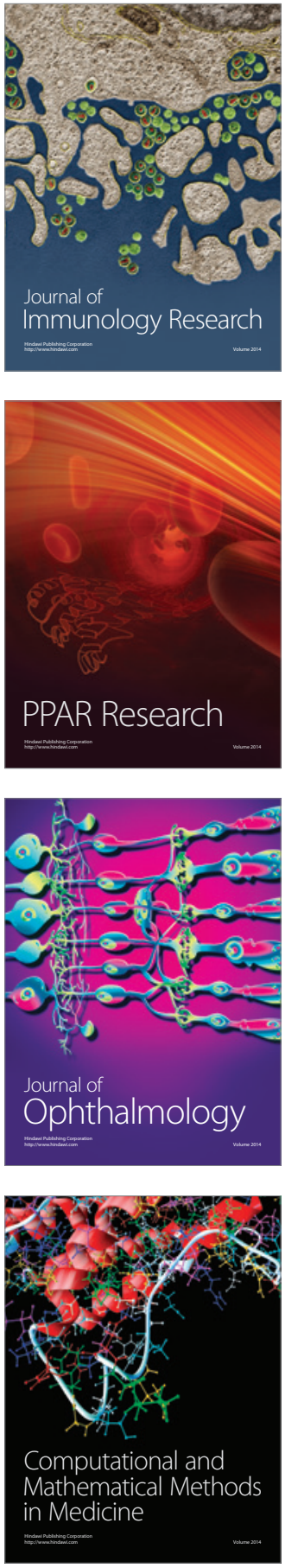

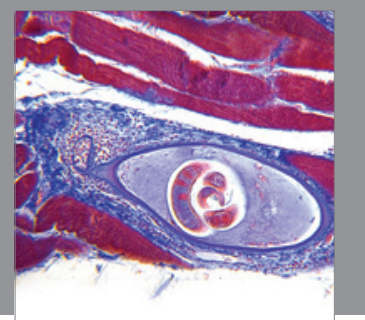

Gastroenterology

Research and Practice
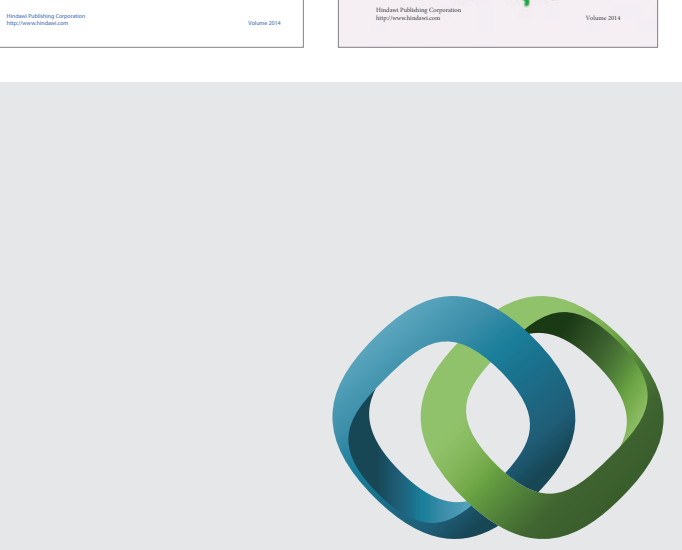

\section{Hindawi}

Submit your manuscripts at

http://www.hindawi.com
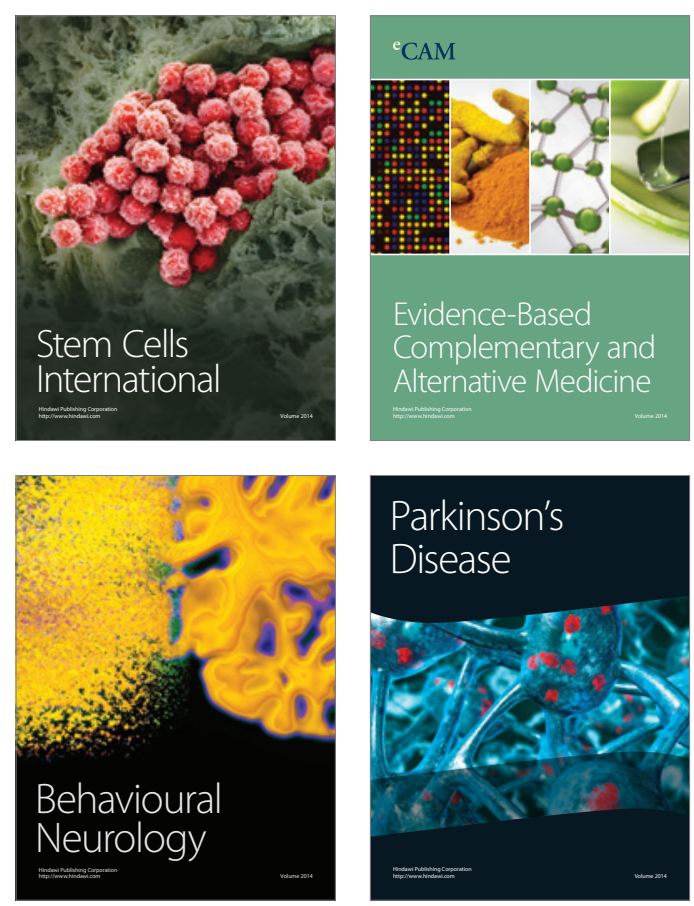

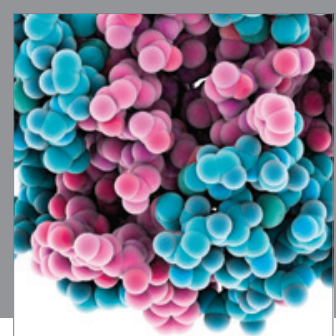

Journal of
Diabetes Research

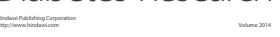

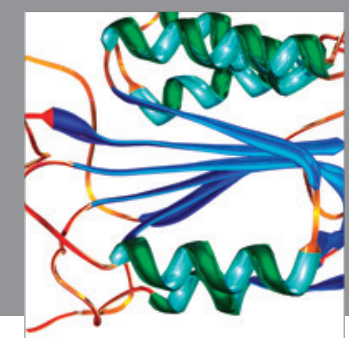

Disease Markers
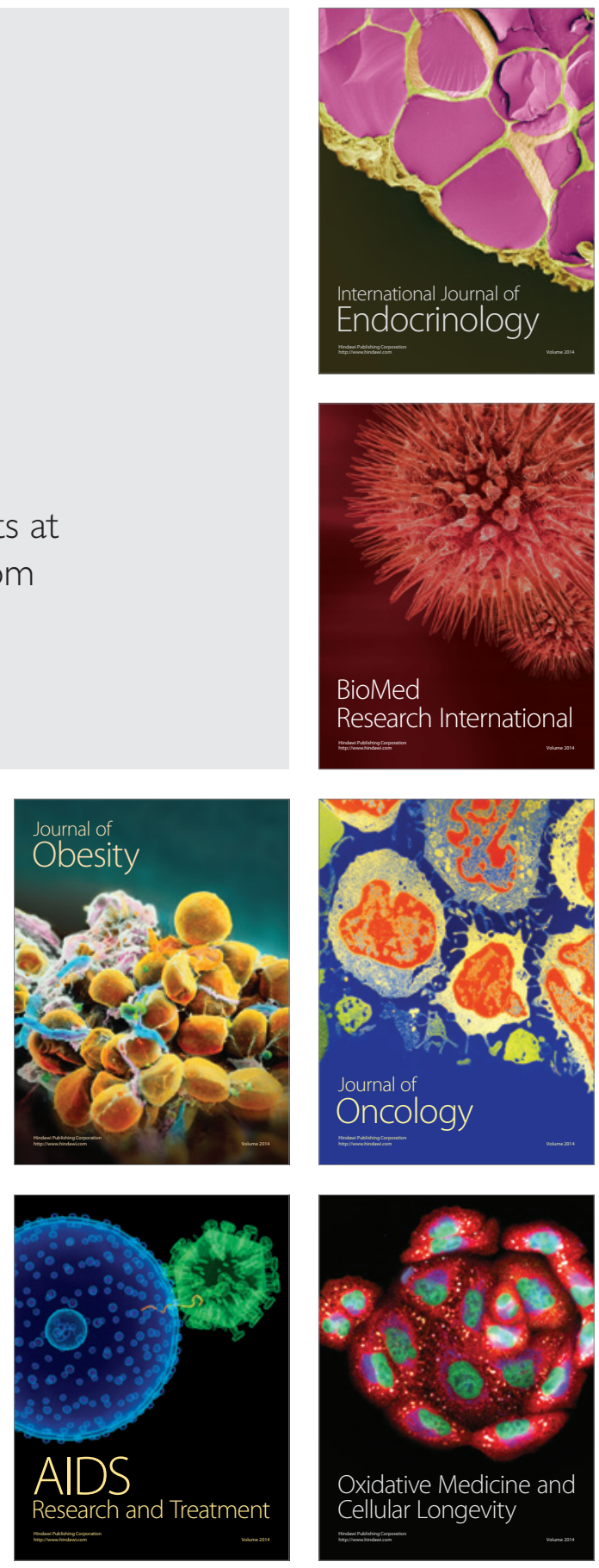